\title{
DIGITALCOMMONS
}

@WAYNESTATE-

Wayne State University

3-1-2008

\section{Failure of Metric Regularity for Major Classes of Variational Systems}

Boris S. Mordukhovich

Wayne State University, boris@math.wayne.edu

\section{Recommended Citation}

Mordukhovich, Boris S., "Failure of Metric Regularity for Major Classes of Variational Systems" (2008). Mathematics Research Reports. Paper 57.

http://digitalcommons.wayne.edu/math_reports/57

This Technical Report is brought to you for free and open access by the Mathematics at DigitalCommons@WayneState. It has been accepted for inclusion in Mathematics Research Reports by an authorized administrator of DigitalCommons@WayneState. 


\title{
FAILURE OF METRIC REGULARITY FOR MAJOR CLASSES OF VARIATIONAL SYSTEMS
}

\author{
BORIS S. MORDUKHOVICH
}

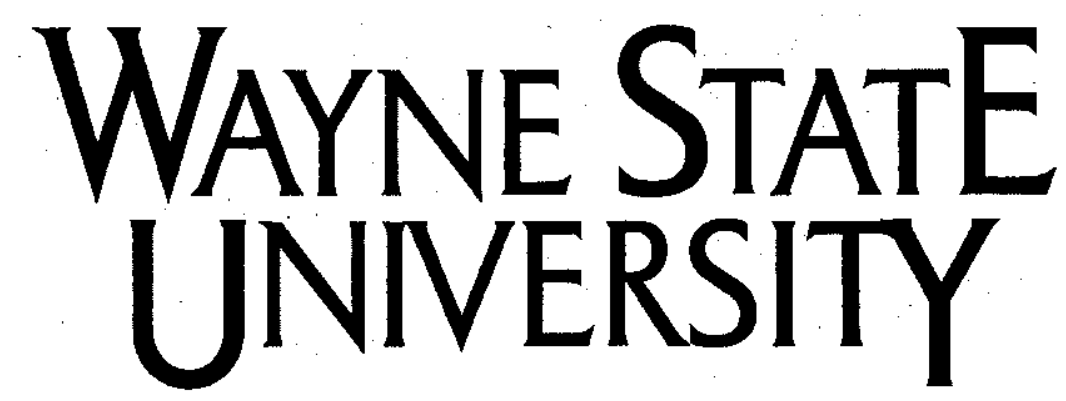

Detroit, MI 48202

Department of Mathematics

Research Report

\section{Series}

\#3

This research was partly supported by the US National Science Foundation and the Australian Research Council 


\title{
FAILURE OF METRIC REGULARITY FOR MAJOR CLASSES OF VARIATIONAL SYSTEMS ${ }^{1}$
}

\author{
BORIS S. MORDUKHOVICH \\ Department of Mathematics, Wayne State University \\ Detroit, MI 48202, USA (email: boris@math.wayne.edu)
}

\section{Dedicated to Professor V. Lakshmikantham in honor of his 85th birthday}

Abstract. The paper is devoted to the study of metric regularity, which is a remarkable property of set-valued mappings playing an important role in many aspects of nonlinear analysis and its applications. We pay the main attention to metric regularity of the socalled parametric variational systems that contain, in particular, various classes of parameterized/perturbed variational and hemivariational inequalities, complementarity systems, sets of optimal solutions and corresponding Lagrange multipliers in problems of parametric optimization and equilibria, etc. Based on the advanced machinery of generalized differentiation, we surprisingly reveal that metric regularity fails for certain major classes of parametric variational systems, which admit conventional descriptions via subdifferentials of convex as well as prox-regular extended-real-valued functions.

Keywords: Variational analysis and optimization; Generalized differentiation; Metric regularity; Lipschitzian properties; Parametric variational systems

\section{Introduction}

It has been well recognized that the property of set-valued mappings known as metric regularity, as well as the linear openness/covering property equivalent to it; play an important role in many aspects of nonlinear and variational analysis and their applications; see, e.g., $[1,3,5,6,8,10,12,13,16]$ with the extensive bibliographies therein. In the afore-mentioned references, the reader can find verifiable conditions ensuring these properties and their implementations in specific situations mainly related to the implicit functions and multifunctions frameworks and to the so-called parametric constraint systems in nonlinear analysis and optimization. The latter class of systems incorporates, in particular, sets of feasible solutions in various constrained optimization and equilibrium problems.

In this paper we pay the main attention to the study of metric regularity for broad classes of parametric variational systems, which include such important objects of variational analysis and optimization as parameterized/perturbed variational

\footnotetext{
${ }^{1}$ Research was partially supported by the US National Science Foundation under grants DMS0304989 and DMS-0603846 and also by the Australian Research Council under grant DP-04511668.
} 
and hemivariational inequalities of different kinds, complementarity systems, sets of optimal solutions and Lagrange multipliers (or Karush-Kuhn-Tucker vectors) in various problems of parametric optimization and equilibria, etc. It comes as a surprise that metric regularity fails for major classes of parametric variational systems including those mentioned above. We demonstrate this by reducing metric regularity to certain Lipschitzian properties of subdifferential mappings generating the variational systems under consideration. The latter properties do not hold for subdifferentials of remarkable classes of extended-real-valued functions, such as convex and prox-regular ones, which are overwhelmingly encountered in variational analysis, optimization, and their applications. Furthermore, metric regularity fails also for nonsubdifferentially generated variational systems described via monotone set-valued operators.

The rest of the paper is organized as follows. In Section 2 we define and discuss the metric regularity and Lipschitzian properties of set-valued mappings considered in this paper. In Section 3 we briefly review generalized differential tools of variational analysis used in the modeling of variational systems and deriving the main results. Section 4 contains descriptions of general parametric variational systems and their most important particular cases of major interest for optimization theory and applications. Finally, in Section 5 we establish the main results on the failure of metric regularity for the major classes of parametric variational systems under consideration.

Throughout the paper we use the standard notation and terminology of variational analysis; cf. $[12,16]$. Unless otherwise stated, $X$ and all the other spaces under consideration are Banach with the norm $\|\cdot\|$ and the canonical pair $\langle\cdot, \cdot\rangle$ between $X$ and its topologically dual space $X^{*}$. Recall that $B B$ stands for the closed unit ball in the space in question, and that we use the symbol $F: X \rightrightarrows Y$ to signify generally a setvalued mapping between $X$ and $Y$ in contrast to a single-valued mapping $f: X \rightarrow Y$. As usual, we denote

$$
\operatorname{dom} F:=\{x \in X \mid F(x) \neq \emptyset\} \text { and } \operatorname{gph} F:=\{(x, y) \in X \times Y \mid y \in F(x)\}
$$

the domain and graph of the mapping $F: X \rightrightarrows Y$, respectively. The inverse mapping $F^{-1}: Y \Rightarrow X$ to $F$ is defined by $F^{-1}(y):=\{x \in X \mid y \in F(x)\}$.

Given a set-valued mapping $F: X \rightrightarrows X^{*}$ between $X$ and $X^{*}$, the symbol

$$
\begin{aligned}
\operatorname{Limsup}_{x \rightarrow \bar{x}} F(x):=\left\{x^{*} \in X^{*} \mid\right. & \exists \text { sequences } x_{k} \rightarrow \dot{\bar{x}}, \quad x_{k}^{*} \stackrel{w^{*}}{\rightarrow} x^{*} \\
& \text { with } \left.x_{k}^{*} \in F\left(x_{k}\right) \text { for all } k \in \mathbb{N}\right\}
\end{aligned}
$$

signifies the sequential Painlevé-Kuratowski outer/upper limit of $F$ at $x \rightarrow \bar{x}$, where $w^{*}$ stands for the weak* topology of $X^{*}$ and where $\mathbb{N}:=\{1,2, \ldots\}$. Given an extendedreal-valued function $\varphi: X \rightarrow \bar{R}:=(-\infty, \infty]$ with the domain

$$
\operatorname{dom} \varphi:=\{x \in X \mid \varphi(x)<\infty\},
$$

the symbol $x \stackrel{\varphi}{\rightarrow} \bar{x} \in \operatorname{dom} \varphi$ indicates that $x \rightarrow \bar{x}$ with $\varphi(x) \rightarrow \varphi(\bar{x})$. 


\section{Metric Regularity and Lipschitzian Properties}

Recall that $F: X \rightrightarrows Y$ is metrically regular around $(\bar{x}, \bar{y}) \in \operatorname{gph} F$ if there are neighborhoods $U$ of $\bar{x}$ and $V$ of $\bar{y}$ and a number $\mu>0$ such that

$$
\operatorname{dist}\left(x ; F^{-1}(y)\right) \leq \mu \operatorname{dist}(y ; F(x)) \text { whenever } x \in U \text { and } y \in V \text {. }
$$

Further, we say that $F: X \Rightarrow Y$ is Lipschitz-like around $(\bar{x}, \bar{y}) \in \operatorname{gph} F$ is there are neighborhoods $U$ of $\bar{x}$ and $V$ of $\bar{y}$ and a number $\ell \geq 0$ such that

$$
F(x) \cap V \subset F(u)+\ell\|x-u\| \mathbb{B} \text { for all } x, u \in U \text {. }
$$

The latter property is also known as the Aubin "pseudo-Lipschitz" property of setvalued mappings; see $[12,16]$. When $V=Y$ in $(2.2)$, it reduces to the classical (Hausdorff) local Lipschitzian property of $F$ around $\tilde{x} \in \operatorname{dom} F$. Note that the Lipschitzian properties under consideration are robust, i.e., stable with respect to small perturbations of the reference points.

It has been well recognized in variational analysis that the metric regularity property of $F$ around $(\bar{x}, \bar{y})$ is equivalent to the Lipschitz-like property of its inverse around $(\bar{y}, \bar{x})$ with the same modulus in (2.1) and (2.2). Similar relationships hold true for certain semilocal and global modifications of the above local metric regularity and Lipschitzian properties and their linear openness/covering counterparts; see, e.g., [12, Section 1.2] with the references and discussions therein.

Observe that the metric regularity and Lipschitzian properties under consideration are defined in primal spaces and are derivative-free, i.e., they do not depend on any derivative-like construction. It turns out nevertheless that, due to variational/extremal principles, they admit complete dual-space characterizations in both finite and infinite dimensions via appropriate coderivatives of set-valued mappings; see [11], [12, Chapter 4], and [16, Chapter 9] with the references and commentaries therein. These constructions and characterizations are not directly employed in what follows, while on their basis we established in [5] the relationships between metric regularity and Lipschitzian properties of parametric variational systems that are crucially used for deriving the main results of this paper in Section 5.

In the next section we briefly review only those constructions and notions of generalized differentiation, which are needed for describing parametric variational systems in Section 4 and establishing the main results presented in Section 5.

\section{Generalized Differentiation}

We start with subdifferential constructions for general extended-real-valued functions, which are used for descriptions of the most interesting classes of variational systems. 
Given an extended-real-valued function $\varphi: X \rightarrow \overline{\mathbb{R}}$ on a Banach space $X$, consider first the $\varepsilon$-subdifferential of $\varphi$ at $x \in \operatorname{dom} \varphi$ defined by

$$
\widehat{\partial}_{\varepsilon} \varphi(x):=\left\{x^{*} \in X^{*} \mid \liminf _{u \rightarrow x} \frac{\varphi(u)-\varphi(x)-\left\langle x^{*}, u-x\right\rangle}{\|u-x\|} \geq-\varepsilon\right\}, \quad \varepsilon \geq 0 .
$$

If $\varepsilon=0$ in (3.1), this construction $\widehat{\partial} \varphi(x):=\widehat{\partial}_{0} \varphi(x)$ is known as Fréchet, or regular, or viscosity subdifferential of $\varphi$ at $\bar{x}$; it remains us the classical Fréchet derivative when the limits exists in (3.1) and equals zero. The latter subdifferential construction is often use in the theory of viscosity solutions to partial differential equations. However, its applications in variational analysis and optimization are rather limited due to the possible emptiness of the subgradient set (3.1) (as, e.g., for $\varphi(x)=-|x|$ at $x=0 \in \mathbb{R}$ ) and due to poor calculus rules available for (3.1). In particular, the sum rule

$$
\widehat{\partial}\left(\varphi_{1}+\varphi_{2}\right)(x) \subset \widehat{\partial} \varphi_{1}(x)+\widehat{\partial} \varphi_{2}(x)
$$

unavoidable in optimization theory and the majority of applications does not hold even in trivial situations as for $\varphi_{1}(x)=|x|$ and $\varphi_{2}(x)=-|x|$.

Significantly better pointwise subdifferential properties are available for the following sequential robust regularization

$$
\partial \varphi(\bar{x}):=\operatorname{Limsup}_{\substack{x \rightarrow \underline{\varphi} \rightarrow \bar{x} \\ \varepsilon \downarrow 0}} \widehat{\partial}_{\varepsilon} \varphi(x)
$$

defined via (1.1) and known as basic/general, or limiting, or Mordukhovich subdifferential of $\varphi$ at $\bar{x} \in \operatorname{dom} \varphi$; see, e.g., the books $[1,7,12,13,14,16,17,18]$ and the references therein for its recent studies and numerous applications in both finitedimensional and infinite-dimensional settings. For convex functions $\varphi: X \rightarrow \overline{\mathbb{R}}$ we have the representation

$$
\partial \varphi(\bar{x})=\left\{x^{*} \in X^{*} \mid\left\langle x^{*}, x-\bar{x}\right\rangle \leq \varphi(x)-\varphi(\bar{x}) \text { whenever } x \in X\right\}
$$

i.e., the subdifferential (3.2) reduces to the classical subdifferential of convex analysis.

Considering a general class of extended-real-valued functions allows us to include sets into the functional framework. Given a set $\Omega \subset X$ and its indicator function $\delta(x ; \Omega)$ equal to 0 for $x \in \Omega$ and $\infty$ otherwise; we define the normal cone to $\Omega$ at $\bar{x} \in \Omega$ by $N(\bar{x} ; \Omega):=\partial \delta(\bar{x} ; \Omega)$. For convex sets $\Omega$ we have the classical normal cone of convex analysis:

$$
N(\bar{x} ; \Omega)=\left\{x^{*} \in X^{*} \mid\left\langle x^{*}, x-\bar{x}\right\rangle \leq 0 \text { whenever } x \in \Omega\right\} .
$$

Particularly good properties of the basic subdifferential (3.2) for general nonconvex functions, including comprehensive calculus rules ("full calculus") have been developed in the framework of Asplund spaces that are Banach spaces in which every 
separable subspace has a separable dual. This class is sufficiently broad including all reflexive Banach spaces as well as those with separable duals; see, e.g., $[1,12]$ for more discussions and references. If $X$ is Asplund and if $\varphi$ is lower semicontinuous (l.s.c.) around $\bar{x}$, the basic subdifferential admits a simplified representation with $\varepsilon=0$ in (3.2). Note that the full calculus for the subdifferential (3.2) and the associated normal and coderivative constructions for sets and set-valued mappings is based on variational/extremal principles of variational analysis; see [12] for comprehensive results in this direction.

Following [16], we say that $\varphi$ is subdifferentially continuous at $\bar{x}$ for some subgradient $\bar{x}^{*} \in \partial \varphi(\bar{x})$ if $\varphi\left(x_{k}\right) \rightarrow \varphi(\bar{x})$ whenever $x_{k} \rightarrow \bar{x}, x_{k}^{*} \stackrel{w^{*}}{\rightarrow} \bar{x}^{*}$ as $k \rightarrow \infty$ with $x_{k}^{*} \in \partial \varphi\left(x_{k}\right)$ for all $k \in \mathbb{N}$. Further, $\varphi$ is prox-regular at $\bar{x} \in \operatorname{dom} \varphi$ for some $\bar{x}^{*} \in \partial \varphi(\bar{x})$ is it is l.s.c. around $\bar{x}$ and there are $\gamma>0$ and $\eta \geq 0$ such that

$$
\begin{gathered}
\varphi(u) \geq \quad(x)+\left\langle\bar{x}^{*}, u-x\right\rangle-\frac{\eta}{2}\|u-x\|^{2} \text { for all } x^{*} \in \partial \varphi(x) \text { with }\left\|x^{*}-\bar{x}^{*}\right\| \leq \gamma \\
\|u-\bar{x}\| \leq \gamma, \quad\|x-\bar{x}\| \leq \gamma, \text { and } \varphi(x) \leq \varphi(\bar{x})+\gamma
\end{gathered}
$$

Various sufficient conditions ensuring the validity of both subdifferential continuity and prox-regularity properties can be found in [16] in the case of $X=\mathbb{R}^{n}$. These properties hold, in particular, for all l.s.c. convex functions as well as for a broad class of strongly amenable functions that play a significant role in variational analysis and its numerous applications.

\section{Parametric Variational Systems}

In this section we consider a class of the so-called parametric variational systems (PVS) described as solution maps to parameterized generalized equations of the type

$$
S(x)=\{y \in Y \mid 0 \in f(x, y)+Q(y)\}
$$

where $f: X \times Y \rightarrow Z$ is a single-valued mapping, while $Q: Y \Rightarrow Z$ is a set-valued mapping between Banach spaces. It has been well recognized that the PVS form (4.1) describes a broad range of moving/parameterized sets associated with optimal solutions and first-order optimality conditions to various problems of parametric optimization and equilibria. For convenience we label $f$ and $Q$ in (4.1) as the base and field mappings, respectively.

Models of this type were introduced and studied by Robinson [15] in the case of $Q(y)=N(y ; \Omega)$, a normal cone mapping to a convex set $\Omega$, when (4.1) describes, due to (3.3), solution maps to parameterized variational inequalities in the form

$$
\text { find } y \in \Omega \text { such that }\langle f(x, y), v-y\rangle \geq 0 \text { for all } v \in \Omega \text {. }
$$


If, in particular, $\Omega$ is a nonnegative orthant of a finite-dimensional space, model (4.2) captures solution maps to parameterized complementarity problems, and hence moving sets of Karush-Kuhn-Tucker (KKT) vectors in parametric problems of nonlinear programming. In general, variational conditions of type (4.1) have been widely accepted as a very useful and convenient framework for the study of a great variety of parametric systems that frequently appear in many aspects of variational analysis, optimization, equilibria, and their numerous applications; see, e.g., the books $[9,12,13,14,16]$ and the references therein.

Variational systems most important for optimization/equilibrium theory and applications mainly relate to generalized equations (4.1) with subdifferential fields when $Q$ is given by a subdifferential/normal cone operator $\partial \varphi$ generated by an extendedreal-valued l.s.c. function $\varphi$, which is often labeled as potential. As mentioned above, this is the case of the classical variational inequalities (4.2) and complementarity problems generated by convex indicator functions $\varphi(\cdot)=\delta(\cdot ; \Omega)$. Formalism (4.1) with $Q=\partial \varphi$ encompasses also other types of variational and extended variational inequalities generated by nonconvex potentials; in particular, the so-called hemivariational inequalities with Lipschitzian potentials.

In this vein we consider, following [12, Chapter 4], two major classes of composite subdifferential PVS. The first one is given in the form

$$
S(x):=\{y \in Y \mid 0 \in f(x, y)+\partial(\psi \circ g)(y)\}
$$

where $g: Y \rightarrow W$ and $f: X \times Y \rightarrow Y^{*}$ are single-valued mappings between Banach spaces, and where $\partial \varphi: Y \rightrightarrows Y^{*}$ is the basic subdifferential mapping (3.2) generated by the composite potential $\varphi=\psi \circ g$ with $\psi: W \rightarrow \overline{\mathbb{R}}$. The afore-mentioned variational systems are particular cases of the composite formalism (4.3).

The second class of composite subdifferential PVS is described by the generalized equations with composite fields

$$
S(x):=\{y \in Y \mid 0 \in f(x, y)+(\partial \psi \circ g)(y)\},
$$

where $g: Y \rightarrow W, \psi: W \rightarrow \bar{R}$, and $f: X \times Y \rightarrow W^{*}$. Formalism (4.4) encompasses, in particular, perturbed implicit complementarity problems of the type: find $y \in Y$ satisfying the relationships

$$
f(x, y) \geq 0, \quad y-g(x, y) \geq 0, \quad\langle f(x, y), y-g(x, y)\rangle=0
$$

where the inequalities are understood in the sense of some order on $Y$.

In the next section we show that the major parametric variational systems of the general type (4.1) with set-valued monotone fields as well as of the composite subdifferential type (4.3) and (4.4) generated by nonsmooth potentials $\psi(\cdot)$ do not exhibit metric regularity. 


\section{Failure of Metric Regularity for Parametric Vari- ational Systems}

This section contains the main results of the paper on the failure of metric regularity for major classes of PVS governed by generalized equations in forms (4.1), (4.3), and (4.4). The results obtained are largely based on the relationships between metric regularity of the PVS under consideration and the Lipschitz-like property of the field mappings in the above generalized equations established in [5].

Let us start with the general model (4.1), where the field mapping $Q: Y \rightrightarrows Y^{*}$ is a monotone operator, i.e., it has the property.

$$
\left\langle y_{1}^{*}-y_{2}^{*}, y_{1}-y_{2}\right\rangle \geq 0 \text { whenever } y_{i}^{*} \in Q\left(y_{i}\right), \quad i=1,2 \text {. }
$$

Recall that a mapping $h: W \rightarrow Z$ between Banach spaces is strictly differentiable at $\bar{w}$ with the derivative $\nabla h(\bar{w}): W \rightarrow Z$ if

$$
\lim _{\substack{w \rightarrow \tilde{w} \\ v \rightarrow \tilde{w}}} \frac{h(w)-h(v)-\nabla h(\bar{w})(w-v)}{\|w-v\|}=0,
$$

which surely holds if $h \in C^{1}$ around $\bar{w}$.

Theorem 5.1 (failure of metric regularity for generalized equations with monotone fields). Let $f: X \times Y \rightarrow Y^{*}$ be a mapping between Asplund spaces that is strictly differentiable at $(\bar{x}, \bar{y})$ with the surjective partial derivative $\nabla_{x} f(\bar{x}, \bar{y})$, and let $Q: Y \rightrightarrows Y^{*}$ be locally closed-graph around $\left(\bar{y}, \bar{y}^{*}\right)$ with $\bar{y}^{*}:=-f(\bar{x}, \bar{y}) \in Q(\bar{y})$. Assume in addition that $Q$ is monotone and that there is no neighborhood of $\bar{y}$ on which $Q$ is single-valued. Then the solution map $S: X \rightrightarrows Y$ in (4.1) is not metrically regular around the reference point $(\bar{x}, \bar{y})$.

Proof. Based on a rather involved coderivative analysis, it is proved in 15 , Theorem 5.6] that the metric regularity of $S$ around $(\bar{x}, \bar{y})$ is equivalent to the Lipschitz-like property of $Q$ around $\left(\bar{y}, \bar{y}^{*}\right)$ under the general assumptions of the theorem (excluding the monotonicity and the local set-valuedness of $Q$ ). On the other hand, it is proved in [2, Proposition 5.1] that the monotonicity of $Q$ and its Lipschitz-like property around $\left(\bar{y}, \ddot{y}^{*}\right)$ imply that the set $Q(y)$ is a singleton for all $y$ in some neighborhood of $\vec{y}$. Thus the opposite assumption imposed in the theorem and the afore-mentioned equivalence ensure that $S$ cannot be metrically regular around $(\bar{x}, \bar{y})$.

Since the set-valuedness of field mappings is a characteristic feature of generalized equations as a satisfactory model to describe variational systems (otherwise they reduce just to standard equations, which that are not of particular interest in the variational framework under consideration), the conclusion of Theorem 5.1 reads that variational systems with monotone fields are not metrically regular under the 
strict differentiability and surjectivity assumptions on base mappings, which do not seem to be restrictive. A major consequence of Theorem 5.1 is the following corollary concerning subdifferential systems with convex potentials, which encompass the classical cases of variational inequalities and complementarity problems in (4.2).

Corollary 5.2 (failure of metric regularity for subdifferential PVS with convex potentials). Let $Q(y)=\partial \varphi(y)$ in (4.1), where $f: X \times Y \rightarrow Y^{*}$ is a mapping between Asplund spaces that is strictly differentiable at $(\bar{x}, \bar{y})$ with the surjective partial derivative $\nabla_{x} f(\bar{x}, \bar{y})$, and where $\varphi: Y \rightarrow \overline{\mathbb{R}}$ is a l.s.c. convex function finite at $\bar{y}$ and such that there is no neighborhood of $\bar{y}$ on which $\varphi$ is Gâteaux differentiable. Then $S$ is not metrically regular around $(\bar{x}, \bar{y})$.

Proof. Observe first that the assumptions imposed on $\varphi$ ensure that the subdifferential mapping $Q(y)=\partial \varphi(y)$ is closed-graph. Furthermore, the classical Rockafellar theorem establishes the maximal monotonicity of the subdifferential mapping $\partial \varphi$ for proper, l.s.c., convex functions on Banach spaces. Thus the conclusion of the corollary follows from the the well-known fact of convex analysis that the subdifferential of such a function is a singleton at the reference point if and only if of the function is Gâteaux differentiable at it.

Note that the classical settings of variational inequalities and complementarity problems in (4.2) correspond to the highly nonsmooth (extended-real-valued) case of the convex indicator functions $Q(y)=\varphi(y)=\delta(y ; \Omega)$ in (4.1). In fact, essentially more general nonconvex subdifferential structures of parametric variational systems prevent the fulfillment of metric regularity for solutions maps (4.1) with no reduction to the field monotonicity; see below.

Let us next consider parametric variational systems with the composite subdifferential structure generated by strongly amenable potentials in (4.3).

Theorem 5.3 (failure of metric regularity for composite subdifferential PVS with strongly amenable potentials). Let $(\bar{x}, \bar{y}) \in \operatorname{gph} S$ for the parametric variational system $S$ given in (4.3), where $g: Y \rightarrow W$ is twice continuously differentiable around $\bar{y}$ with the surjective derivative $\nabla g(\bar{y})$, where $f: X \times Y \rightarrow Y^{*}$ is strictly differentiable at $(\bar{x}, \bar{y})$ with the surjective partial derivative $\nabla_{x} f(\bar{x}, \bar{y})$, and where all the spaces under consideration are Asplund. Set $\bar{w}:=g(\bar{y})$ and assume that $\psi: W \rightarrow \bar{R}$ is a l.s.c. convex function finite at $\bar{w}$ and such that there is no neighborhood of $\bar{w}$ on which $\psi$ is Gâteaux differentiable. Then $S$ is not metrically regular around $(\bar{x}, \bar{y})$.

Proof. Observe that, according to [16, Definition 10.23], the potential $\varphi=\psi \circ g$ is strongly amenable around $\bar{y}$ and that the subdifferential mapping $\partial \psi: W \rightrightarrows W^{*}$ is closed-graph due the assumptions imposed on $\psi$. Since all the requirement of $[5$, Theorem 5.7] are met, we conclude that the metric regularity of $S$ around $(\bar{x}, \bar{y})$ is 
equivalent to the Lipschitz-like property of $\partial \psi$ around $(\bar{w}, \bar{v})$, where $\bar{v} \in \partial \psi(\bar{w})$ is uniquely determined by $\nabla g(\bar{y})^{*} \bar{v}=-f(\bar{x}, \bar{y})$. As shown in the proofs of Theorem 5.1 and Corollary 5.2 , the afore-mentioned Lipschitz-like property of $\partial \psi$ does not hold under the assumptions made.

The next result establishes a similar conclusion on the failure of local metric regularity for the subdifferential PVS (4.4) with composite fields.

Theorem 5.4 (failure of metric regularity for subdifferential PVS with composite fields and convex potentials). Let $(\vec{x}, \bar{y}) \in \operatorname{gph} S$ for $S$ given in (4.4), where $g: Y \rightarrow W$ is strictly differentiable at $\bar{y}$ with the surjective derivative $\nabla g(\bar{y})$, where $f: X \times Y \rightarrow W^{*}$ is strictly differentiable at $(\tilde{x}, \bar{y})$ with the surjective partial derivative $\nabla_{x} f(\bar{x}, \bar{y})$, and where all the spaces under consideration are Asplund. Assume in addition that $\psi: W \rightarrow \overline{\mathbb{R}}$ is a l.s.c. convex function finite at $\bar{w}=g(\bar{y})$ and such that there is no neighborhood of $\bar{w}$ on which $\psi$ is Gâteaux differentiable. Then $S$ is not metrically regular around the reference point $(\bar{x}, \bar{y})$.

Proof. It is easy to observe that the assumptions made ensure that the composite mapping $\partial \psi \circ g$ is closed graph around $(\bar{y},-f(\bar{x}, \bar{y}))$. By [5, Theorem 5.8] we have, under the general assumptions of this theorem, that the metric regularity of $S$ around $(\bar{x}, \bar{y})$ is equivalent to the Lipschitz-like property of the subdifferential mapping $\partial \psi$ around $(\bar{w}, \bar{z})$ with $\bar{z}=-f(\bar{x}, \bar{y})$. The latter property does not hold under the assumptions imposed on $\psi$ in the theorem due to the arguments given in the proofs of Theorem 5.1 and Corollary 5.2.

The next two theorems concern subdifferential variational systems (4.3) and (4.4) involving nonconvex functions $\psi$ therein. We replace the above convexity assumption by the prox-regularity of $\psi$ defined on a finite-dimensional space $W$.

Theorem 5.5 (failure of metric regularity for composite subdifferential PVS with prox-regular potentials). Let $(\bar{x}, \bar{y}) \in \operatorname{gph} S$ for the parametric variational system $S$ given in (4.3), where $g: Y \rightarrow \mathbb{R}^{n}$ is twice continuously differentiable around $\bar{y}$ with the surjective derivative $\nabla g(\bar{y})$, where $f: X \times Y \rightarrow Y^{*}$ is strictly differentiable at $(\bar{x}, \bar{y})$ with the surjective partial derivative $\nabla_{x} f(\bar{x}, \bar{y})$, and where the spaces $X, Y$, and $Y^{*}$ are Asplund. Set $\bar{w}:=g(\bar{y})$ and assume in a.ddition that:

(i) either $\psi$ is locally Lipschitzian around $\bar{w}$,

(ii) or $\psi$ is prox-regular and subdifferential continuous at $\bar{w}$ for the subgradient $\bar{v} \in \partial \psi(\bar{w})$, which is uniquely determined by $\nabla g(\bar{y})^{*} \bar{v}=-f(\bar{x}, \bar{y})$.

Then $S$ is not metrically regular around $(\bar{x}, \bar{y})$ provided that there is no neighborhood of $\bar{w}$ on which $\psi$ is Gâteaux differentiable.

Proof. It is proved in [5, Theorem 5.7] that the metric regularity property of $S$ around $(\bar{x}, \bar{y})$ is equivalent to the Lipschitz-like property of the basic subdifferential mapping 
$\partial \psi$ around $(\bar{w}, \bar{v})$, for $\bar{w}$ and $\bar{v} \in \partial \psi(\bar{w})$ defined in the theorem, provided that $\partial \psi$ is locally closed-graph around $(\bar{w}, \bar{v})$ with no additional assumptions imposed in (i) and (ii). It is easy to check that the assumptions made in both cases (i) and (ii) ensure the afore-mentioned closed-graph property of $\partial \psi$ around $(\bar{w}, \bar{v})$. Furthermore, the main result of [4] establishes that in both cases (i) and (ii) the Lipschitz-like property of $\partial \psi$ around $(\bar{w}, \bar{v})$ yields that $\psi$ is in fact $C^{1,1}$ around $\bar{w}$, i.e., it is continuously Fréchet (and hence Gâteaux) differentiable and its derivative is locally Lipschitzian around this point. Thus the solution map $S$ cannot be metrically regular around the reference point $(\bar{x}, \bar{y})$ under the assumptions imposed in the theorem.

The final result of the paper concerns metric regularity of PVS (4.4) with composite fields in the case of prox-regular potentials $\psi$ therein.

Theorem 5.6 (failure of metric regularity for subdifferential PVS with composite fields and prox-regular potentials). Let $(\bar{x}, \bar{y}) \in \operatorname{gph} S$ for $S$ defined by (4.4), where $g: Y \rightarrow \mathbb{R}^{n}$ is strictly differentiable at $\bar{y}$ with the surjective derivative $\nabla g(\bar{y})$, where $f: X \times Y \rightarrow \mathbb{R}^{n}$ is strictly differentiable at $(\bar{x}, \bar{y})$ with the surjective partial derivative $\nabla_{x} f(\bar{x}, \bar{y})$, and where the spaces $X$ and $Y$ are Asplund. Set $\bar{w}:=g(\bar{y})$ and assume in addition that either (i) or (ii) of Theorem 5.5 is satisfied, and that there is no neighborhood of $\bar{w}$ on which $\psi$ is Gâteaux differentiable. Then the solution map $S$ is not metrically regular around $(\bar{x}, \bar{y})$.

Proof. We can directly check that the assumptions made in the theorem ensure that the composition $\partial \psi \circ g$ is closed-graph around the point $(\bar{y},-f(\bar{x}, \bar{y}))$. Employing [5, Theorem 5.8], we conclude that the metric regularity of $S$ around $(\bar{x}, \bar{y})$ is equivalent to the Lipschitz-like property of $\partial \psi$ around $(\bar{w}, \bar{v})$, where $\bar{v}$ is defined in (ii) of Theorem 5.5. By [4], the latter yields in both cases (i) and (ii) that $\psi$ must be $C^{1,1}$ around $\bar{w}$. Thus the solution map $S$ in (4.4) fails to exhibit the metric regularity property around $(\bar{x}, \bar{y})$ if all the assumptions of the theorem are satisfied.

\section{References}

[1] J.M. Borwein, Q.J. Zhu, Techniques of Variational Analysis, Springer, Berlin, 2005.

[2] A.L. Dontchev, W.W. Hager, Implicit functions, Lipschitz maps and stability in optimization, Math. Oper. Res. 19 (1994), 753-768.

[3] A.L. Dontchev, A.S. Lewis, R.T. Rockafellar, The radius of metric regularity, Trans. Amer. Math. Soc. 355 (2003), 493-517.

[4] A. Eberhard, B.S. Mordukhovich, C.E.M. Pearce, On differentiability properties of prox-regular functions, preprint (2008).

[5] W. Geremew, B.S. Mordukhovich, N.M. Nam, Coderivative calculus and metric regularity for constraint and variational systens, Nonlinear Anal., to appear. 
[6] A.D. Ioffe, Metric regularity and subdifferential calculus, Russian Math. Surveys 55 (2000), 501-558.

[7] V. Jeyakumar and D.T. Luc, Nonsmooth Vector Functions and Continuous Optimization, Springer, New York, 2008.

[8] A. Jourani and L. Thibault, Coderivative of multivalued mappings, locally compact cones and metric regularity, Nonlinear Anal. 35 (1999), 925-945.

[9] F. Facchinei, J.-P. Pang, Finite-Dimensional Variational Inequalities and Complementarity Problems, Springer, New York, 2003.

[10] D. Klatte and B. Kummer, Nonsmooth Equations in Optimization: Regularity, Calculus, Methods, and Applications, Kluwer Academic Publishers, Boston, 2002.

[11] B.S. Mordukhovich, Complete characterizations of openness, metric regularity, and Lipschitzian properties of multifunctions, Trans. Amer. Math. Soc. 340 (1993), 1-35.

[12] B.S. Mordukhovich, Variational Analysis and Generalized Differentiation, I: Basic Theory, Springer, Berlin, 2006.

[13] B.S. Mordukhovich, Variational Analysis and Generalized Differentiation, II: Applications, Springer, Berlin, 2006

[14] J.V. Outrata, Mathematical programs with equilibrium constraints: theory and numerical methods, in: J. Haslinger, G.E. Stavroulakis (Eds.), Nonsmooth Mechanics of Solids, CISM Cources and Lecture Notes, No. 485, Springer, New York, 2006, pp. 221-274.

[15] S.M. Robinson, Generalized equations and their solutions, I: basic theory, Math. Prog. Study 10 (1979), 128-141.

[16] R.T. Rockafellar, R.J-B. Wets, Variational Analysis, Springer; Berlin, 1998.

[17] W. Schirotzek, Nonsmooth Analysis, Springer, Berlin, 2007.

[18] R.B. Vinter, Optimal Control, Birkhäuser, Boston, 2000. 